\title{
CRANIO-CEREBRAL INJURIES AS THE CAUSE OF HOSPITALIZATIONS IN A PEDIATRIC INTENSIVE CARE UNIT IN A WARSAW HOSPITAL DURING 2005-2009
}

\author{
A. Nitsch-Osuch ${ }^{1}$, E. Kuchar ${ }^{2}$, A. Drosio ${ }^{3}, K_{\text {K. Zycinska }}{ }^{1}$, K. Wardyn ${ }^{1}$ \\ ${ }^{1}$ Department of Family Medicine, Warsaw Medical University, Warsaw, Poland, \\ anitsch@amwaw.edu.pl; \\ 2Department of Pediatric Infectious Diseases, Wroclaw Medical University, Wroclaw, \\ Poland, ekuchar@gmail.com; \\ ${ }^{3}$ Academy of Economics and Humanities, Warsaw, Poland, adrosio@wp.pl
}

Background: Cranio-cerebral injuries are the most common reasons of hospitalizations in pediatric intensive care units, following cardiovascular disorders and neoplasmas. The type of cranio-cerebral injury and circumstances leading to the injury strictly depend on the age of a victim.

Objectives: To estimate the proportion of children with cranio-cerebral injuries among patients hospitalized in one pediatric intensive care unit in Warsaw.

Materials and methods: The retrospective analysis of medical documentation of patients aged 0-18 years hospitalized with diagnosis of cranio-cerebral injury in a pediatric intensive care unit of one of tertiary hospitals in Warsaw in 2005-2009.

Results: 195 patients with cranio-cerebral injury were hospitalized, which makes up 21.6\% of all hospitalized patients. Most of the patients with cranio-cerebral injury were boys (56\%) and most of them were aged 13-18 years (38\%). The majority of children with craniocerebral injuries were victims of traffic accidents (51.4\%). The mortality rate among children with cranio-cerebral injury was $24.5 \%$, only 3 patients became organ donors. A minority of patients (41\%) with cranio-cerebral injury was in a good condition and with complete contact after the discharge from the intensive care unit.

Conclusions: Cranio-cerebral injuries plays an important role in hospitalizations among patients in pediatric intensive care unit. Some of them could have been avoided thanks to the better care and awareness of both children and their caregivers. More educational activities directed to the society are needed. General practitioners may and should be more involved into the educational activities and prophylactic procedures in order to diminish number of cranio-cerebral injuries in children and adolescents. Medical professional must also know and remember that some of the cerebro-cranial injuries may be a result of domestic violence. 